\title{
Coherency in Neutrino-Nucleus Elastic Scattering
}

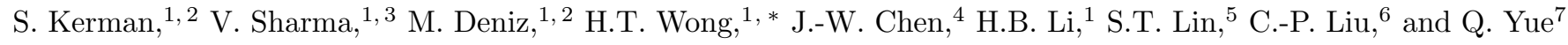 \\ (TEXONO Collaboration) \\ 1 Institute of Physics, Academia Sinica, Taipei 11529, Taiwan. \\ 2 Department of Physics, Dokuz Eylül University, Buca, Izmir 35160, Turkey. \\ 3 Department of Physics, Banaras Hindu University, Varanasi 221005, India. \\ 4 Department of Physics, CTS and LeCosPA, National Taiwan University, Taipei 10617, Taiwan. \\ 5 College of Physical Science and Technology, Sichuan University, Chengdu 610064, China. \\ 6 Department of Physics, National Dong Hwa University, Shoufeng, Hualien 97401, Taiwan. \\ ${ }^{7}$ Department of Engineering Physics, Tsinghua University, Beijing 100084, China.
}

(Dated: March 4, 2018)

\begin{abstract}
Neutrino-nucleus elastic scattering provides a unique laboratory to study the quantum mechanical coherency effects in electroweak interactions, towards which several experimental programs are being actively pursued. We report results of our quantitative studies on the transitions towards decoherency. A parameter $(\alpha)$ is identified to describe the degree of coherency, and its variations with incoming neutrino energy, detector threshold and target nucleus are studied. The ranges of $\alpha$ which can be probed with realistic neutrino experiments are derived, indicating complementarity between projects with different sources and targets. Uncertainties in nuclear physics and in $\alpha$ would constrain sensitivities in probing physics beyond the standard model. The maximum neutrino energies corresponding to $\alpha>0.95$ are derived.
\end{abstract}

PACS numbers: $13.15 .+\mathrm{g}, 03.65 .-\mathrm{w}, 21.10 . \mathrm{Ft}$

The elastic scattering of a neutrino with a nucleus [1, 2]

$$
\nu \mathrm{A}_{e l}: \quad \nu+A(Z, N) \rightarrow \nu+A(Z, N),
$$

where $A(Z, N)$ denotes the atomic nucleus with its respective atomic, charge and neutron numbers, is a fundamental electroweak neutral current process in the Standard Model (SM) which has never been experimentally observed. It can provide a sensitive probe to physics beyond SM (BSM) [3, 4] and plays an important role in astrophysical processes [1, 5. It offers prospects to study neutron density distributions [6], to detect supernova neutrinos 7 and to provide a compact and transportable neutrino detector for real-time monitoring of nuclear reactors 8 . The $\nu \mathrm{A}_{e l}$ events from solar and atmospheric neutrinos are the irreducible background [9] to forthcoming generation of dark matter experiments [10]. There are active experimental programs to observe and measure the processes with neutrinos from reactors [1] or from decay-at-rest pions (DAR- $\pi$ ) 4] with a spallation neutron source 12 .

The $\nu \mathrm{A}_{e l}$ reaction provides a unique laboratory to study the quantum mechanical coherency effects in electroweak interactions. At low momentum transfer, the de Broglie wavelength of the neutrinos is large compared with the nucleus, and the scattering amplitude of individual nucleons will coherently add to contribute to the cross-sections. Typically the neutrino energy $\left(E_{\nu}\right)$ and the measurable nuclear recoil kinetic energy $(T)$ are much less than the target nucleus mass $(M)$ in the discussion of coherency. The three-momentum transfer

\footnotetext{
*Corresponding Author: htwong@phys.sinica.edu.tw
}

$(q \equiv|\vec{q}|)$ is given by $q^{2}=2 M T+T^{2} \simeq 2 M T$. Kinematics places constraints to the maximum recoil energy to be $T_{\max }=2 E_{\nu}{ }^{2} /\left(M+2 E_{\nu}\right) \simeq 2 E_{\nu}{ }^{2} / M$.

A generic scale of $E_{\nu}<50 \mathrm{MeV}$ is usually taken to characterize the requirement of coherency. The theme of this article is to quantify this transition - the first such investigation in the literature. We parametrize the degree of coherency and study its dependence on $E_{\nu}$, nuclear recoil detection threshold $\left(T_{\min }\right)$, and the nucleus $A(Z, N)$. Potential reaches of the up-coming experimental programs are derived.

The differential cross-section of $\nu \mathrm{A}_{e l}$ scattering in $\mathrm{SM}$ is given by [2]:

$$
\begin{aligned}
\frac{d \sigma_{\nu \mathrm{A}_{e l}}}{d q^{2}}\left(q^{2}, E_{\nu}\right)= & \frac{1}{2}\left[\frac{G_{F}^{2}}{4 \pi}\right]\left[1-\frac{q^{2}}{4 E_{\nu}{ }^{2}}\right] \\
& {\left[\varepsilon Z F_{Z}\left(q^{2}\right)-N F_{N}\left(q^{2}\right)\right]^{2} }
\end{aligned}
$$

or, equivalently, in the experimental measurable $T$ as

$$
\frac{d \sigma_{\nu \mathrm{A}_{e l}}}{d T}=2 M\left[\frac{d \sigma_{\nu \mathrm{A}_{e l}}}{d q^{2}}\right]
$$

where $F_{Z}\left(q^{2}\right)$ and $F_{N}\left(q^{2}\right)$ are, respectively, the proton and neutron nuclear form factors for $A(Z, N)$, while $\varepsilon \equiv\left(1-4 \sin ^{2} \theta_{\mathrm{W}}\right)=0.045$, indicating the dominant contributions are from the neutrons. The total cross-section depends on $\left(E_{\nu}, T_{\min } ; M, Z, N\right)$ and is given by:

$$
\sigma_{\nu \mathrm{A}_{e l}}=\int_{q_{\min }^{2}}^{q_{\max }^{2}}\left[\frac{d \sigma_{\nu \mathrm{A}_{e l}}}{d q^{2}}\left(q^{2}, E_{\nu}\right)\right] d q^{2}
$$

where the integration limits of $q_{\max }^{2}=4 E_{\nu}{ }^{2}\left[M /\left(M+2 E_{\nu}\right)\right] \simeq 4 E_{\nu}{ }^{2} \quad$ and $\quad q_{\min }^{2}=2 M T_{\min }$ 
(a)

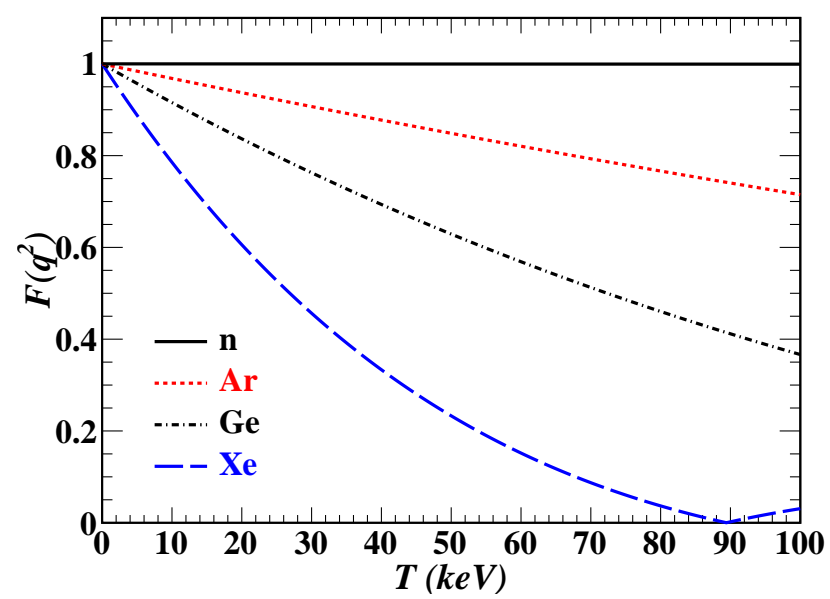

(b)

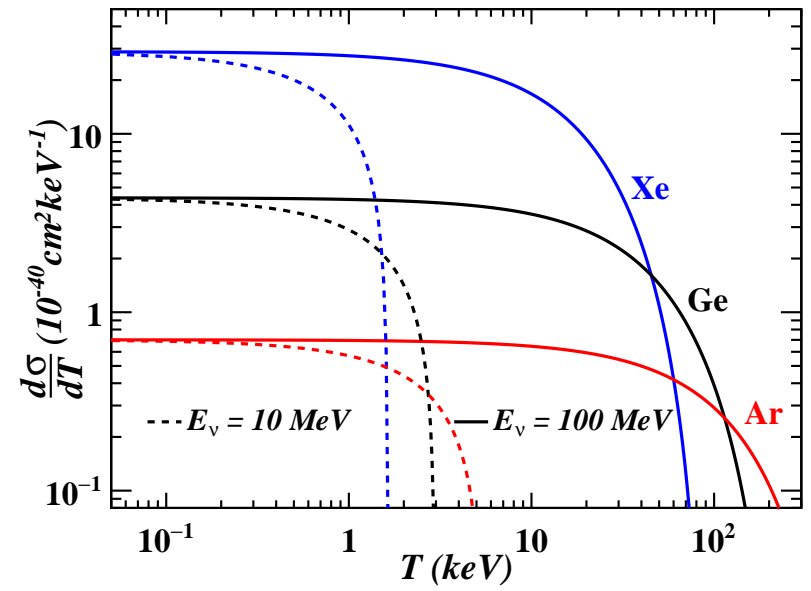

(c)

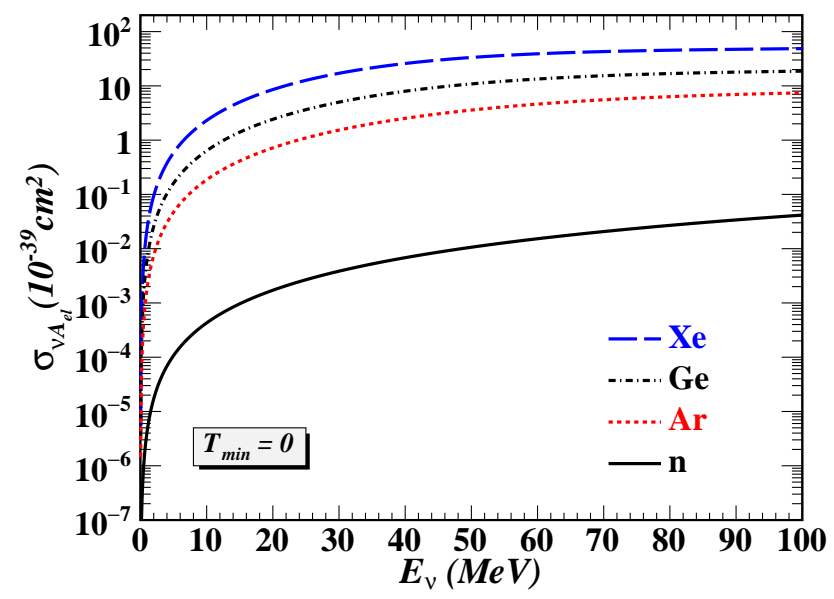

FIG. 1: (a) nuclear form factor $F\left(q^{2}\right)$ as function of $T$, related by $q^{2}=2 M T$; (b) differential cross-section of $\nu \mathrm{A}_{e l}$ as function of $T$ at $E_{\nu}=(10,100) \mathrm{MeV}$; and (c) total cross-section $\left(\sigma_{\nu \mathrm{A}_{e l}}\right)$ at $T_{\min }=0$ as function of $E_{\nu}$. The nuclei (n,Ar,Ge,Xe) are selected for illustrations.
TABLE I: Illustrations at $E_{\nu}=50 \mathrm{MeV}-$ the maximum nuclear recoil energy, the corresponding lower bounds of $F\left(q^{2}\right)$, as well as the coherency factors $\alpha$ and $\xi$ at $T_{\text {min }}=0$.

\begin{tabular}{ccccc}
\hline \hline & $\mathrm{n}$ & $\mathrm{Ar}$ & $\mathrm{Ge}$ & $\mathrm{Xe}$ \\
\hline$T_{\max }(\mathrm{keV})$ & 4810 & 134 & 73.8 & 40.9 \\
$F\left(q^{2}\right)>$ & 0.97 & 0.63 & 0.49 & 0.32 \\
$\alpha>$ & - & 0.77 & 0.68 & 0.57 \\
$\xi>$ & - & 0.78 & 0.69 & 0.58 \\
\hline \hline
\end{tabular}

are defined by the kinematics and detection threshold, respectively. A threshold of $T_{\min }<2 E_{\nu}{ }^{2} / M$ is required to detect neutrinos of energy $E_{\nu}$.

Various aspects in the calculations of the nuclear form factors of Eq. 2 are recently discussed in Ref. [2]. We adopt the effective method of Ref. [13] which assumes the same form factors for neutrons and protons: $F_{Z}\left(q^{2}\right)=F_{N}\left(q^{2}\right) \equiv F\left(q^{2}\right) \in[0,1]$, with

$$
F\left(q^{2}\right)=\left[\frac{3}{q R_{0}}\right] J_{1}\left(q R_{0}\right) \exp \left[-\frac{1}{2} q^{2} s^{2}\right],
$$

where $J_{1}(x)$ is the first-order spherical Bessel function. The target nuclei dependence is introduced through $R_{0}^{2}=R^{2}-5 s^{2}, s=0.5 \mathrm{fm}$ and $R=1.2 A^{\frac{1}{3}} \mathrm{fm}$. An alternative derivation [6] gives form factors consistent to $<0.07(1.7) \%$ within the kinematic ranges corresponding to $E_{\nu}=10(50) \mathrm{MeV}$.

Several nuclei with experimental interest and having different mass ranges - (neutron, Ar, Ge,Xe) at $Z=(0,18,32,54)-$ are selected for studies. (CsI, having $Z=55$ and 53 , can be approximated as Xe in this discussion). Their corresponding differential and total cross-sections are the averages of Eqs. 38 4 due to individual isotopes weighted by their respective isotopic-abundances. The nuclear form factors $F\left(q^{2}\right)$ as function of $T$, the differential cross-sections at fixed $E_{\nu}=(10,100) \mathrm{MeV}$ and the total cross-sections at $T_{\min }=0$ are depicted in Figures 1 a,b\&c, respectively. The $F\left(q^{2}\right)$ ranges at $E_{\nu}=50 \mathrm{MeV}$ are illustrated in TableI. The nuclear effects as characterized by the deviations from unity are significant for heavy nuclei.

At $q^{2} \rightarrow 0$ and $F\left(q^{2}\right) \simeq 1$, full coherency is achieved when the scattering amplitudes due to individual nucleons are perfectly aligned and are summed with no relative phase angle, such that the total cross-section is maximal. In particular at $T_{\min }=0$,

$$
\sigma_{\nu \mathrm{A}_{e l}}\left(T_{\min }=0\right)=\frac{G_{F}^{2} E_{\nu}^{2}}{4 \pi}[\varepsilon Z-N]^{2} .
$$

The experimental signature of full coherency is that $\sigma_{\nu \mathrm{A}_{e l}}$ varies as $[\varepsilon Z-N]^{2}$. Another feature is that the differential cross-section at small $\mathrm{T}$,

$$
\frac{d \sigma_{\nu \mathrm{A}_{e l}}}{d T}(T \rightarrow 0) \simeq\left[\frac{G_{F}^{2} M}{4 \pi}\right][\varepsilon Z-N]^{2}
$$

varies with the same factor and is independent of $E_{\nu}$, as depicted in Figure 1 $\mathrm{b}$. 
We note that "coherent pion production" [14] with accelerator neutrinos at high energy is a distinctly different process. The coherency is due to the coupling of a virtual meson with the nucleus producing a physical pion, and hence is a strong interaction effect and varies as $A^{2}$. The coherency in $\nu \mathrm{A}_{e l}$ interactions, on the other hand, is due to the coupling of a virtual Z-boson with the nucleus, and hence is an electroweak process.

Departure from coherency for $\sigma_{\nu \mathrm{A}_{e l}}$ is characterized by deviations from the $[\varepsilon Z-N]^{2}$ scaling as $q^{2}$ increases. The amplitude vectors of the different nucleons would add with a finite relative phase angle rather than being perfectly aligned. The combined amplitude $\mathcal{A}$ can be described by

$$
\mathcal{A}=\sum_{j=1}^{Z} e^{i \theta_{j}} \mathcal{X}_{j}+\sum_{k=1}^{N} e^{i \theta_{k}} \mathcal{Y}_{k}
$$

where $\mathcal{X}_{j}\left(\mathcal{Y}_{k}\right)$ denotes the coupling strength while $e^{i \theta_{j}}\left(e^{i \theta_{k}}\right)$ is the phase for protons(neutrons). For electroweak processes such as $\sigma_{\nu \mathrm{A}_{e l}},\left(\mathcal{X}_{j}, \mathcal{Y}_{k}\right)=(-\varepsilon, 1)$.

The cross-section comprises $(N+Z)^{2}$ terms:

$$
\begin{aligned}
\sigma_{\nu \mathrm{A}_{e l}} \propto & \mathcal{A} \mathcal{A}^{\dagger} \\
= & \sum_{j=1}^{Z} \mathcal{X}_{j}^{2}+\sum_{k=1}^{N} \mathcal{Y}_{k}^{2} \\
& +\sum_{j=l+1}^{Z} \sum_{l=1}^{Z-1}\left[e^{i\left(\theta_{j}-\theta_{l}\right)}+e^{-i\left(\theta_{j}-\theta_{l}\right)}\right] \mathcal{X}_{j} \mathcal{X}_{l} \\
& +\sum_{k=m+1}^{N} \sum_{m=1}^{N-1}\left[e^{i\left(\theta_{k}-\theta_{m}\right)}+e^{-i\left(\theta_{k}-\theta_{m}\right)}\right] \mathcal{Y}_{k} \mathcal{Y}_{m} \\
& +\sum_{j=1}^{Z} \sum_{k=1}^{N}\left[e^{i\left(\theta_{j}-\theta_{k}\right)}+e^{-i\left(\theta_{j}-\theta_{k}\right)}\right] \mathcal{X}_{j} \mathcal{Y}_{k}
\end{aligned}
$$

Assuming that the decoherence effects between any nucleon pairs can be described by the average phase misalignment angle $\langle\phi\rangle \in[0, \pi / 2]$, it follows that

$$
\left[e^{i\left(\theta_{j}-\theta_{k}\right)}+e^{-i\left(\theta_{j}-\theta_{k}\right)}\right]=2 \cos \left(\theta_{j}-\theta_{k}\right)=2 \cos \langle\phi\rangle
$$

and identically for the other subscript pairs in Eq. 9 with $(j, k) \leftrightarrow(j, l)$ and $(k, m)$. The degree of coherency can therefore be quantified by a measurable parameter $\alpha$, defined as $\alpha \equiv \cos \langle\phi\rangle \in[0,1]$.

The cross-section ratio between $A(Z, N)$ and neu$\operatorname{tron}(0,1)$, following expansion of Eq. 9 and assignment of $\left(\mathcal{X}_{j}, \mathcal{Y}_{k}\right)$, is given by:

$$
\begin{aligned}
& \frac{\sigma_{\nu \mathrm{A}_{e l}}(Z, N)}{\sigma_{\nu \mathrm{A}_{e l}}(0,1)} \\
= & \left\{\varepsilon^{2} Z+N+\varepsilon^{2} Z(Z-1) \alpha+N(N-1) \alpha-2 \varepsilon Z N \alpha\right\} \\
= & \left\{Z \varepsilon^{2}[1+\alpha(Z-1)]+N[1+\alpha(N-1)]-2 \alpha \varepsilon Z N\right\} .
\end{aligned}
$$

The limiting conditions are: (a) $\alpha=1$ implies full coherency or $\sigma_{\nu \mathrm{A}_{e l}} \propto[\varepsilon Z-N]^{2}$, while (b) $\alpha=0$ brings total decoherency or $\sigma_{\nu \mathrm{A}_{e l}} \propto\left[\varepsilon^{2} Z+N\right]$. (a)

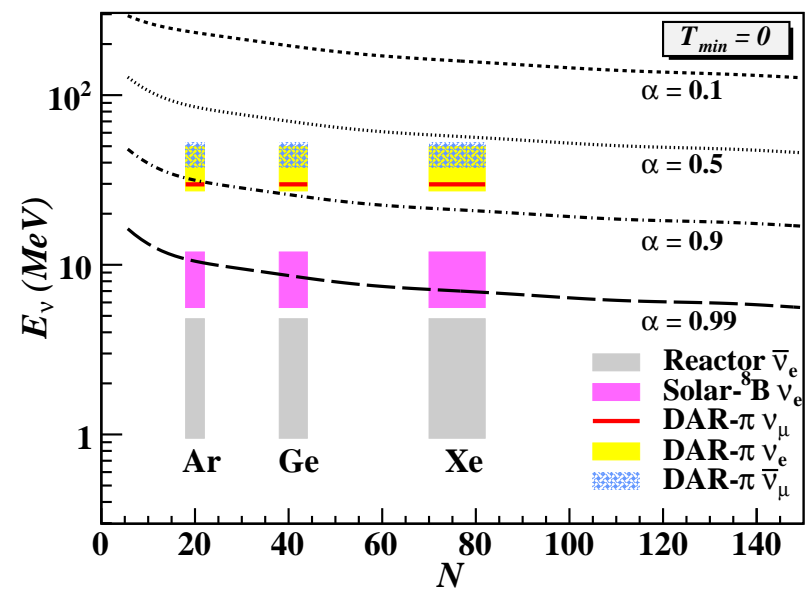

(b)

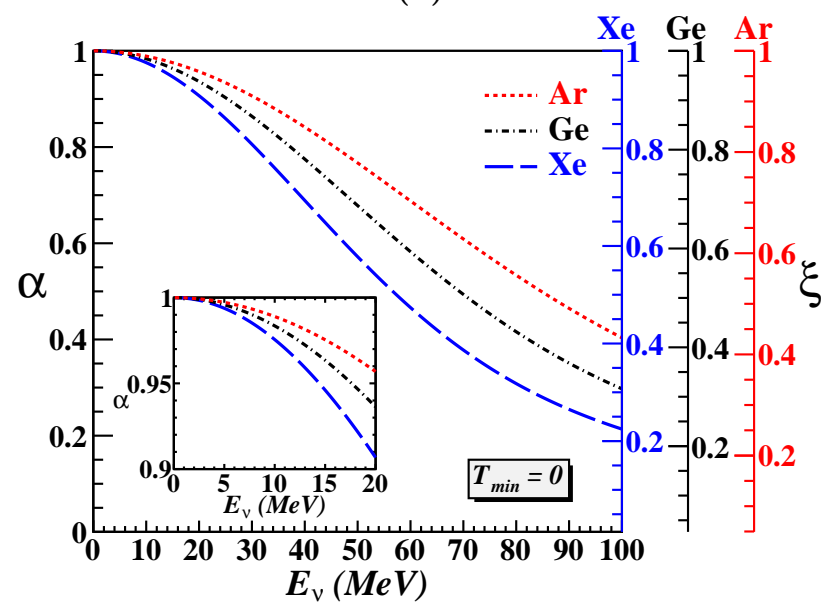

(c)

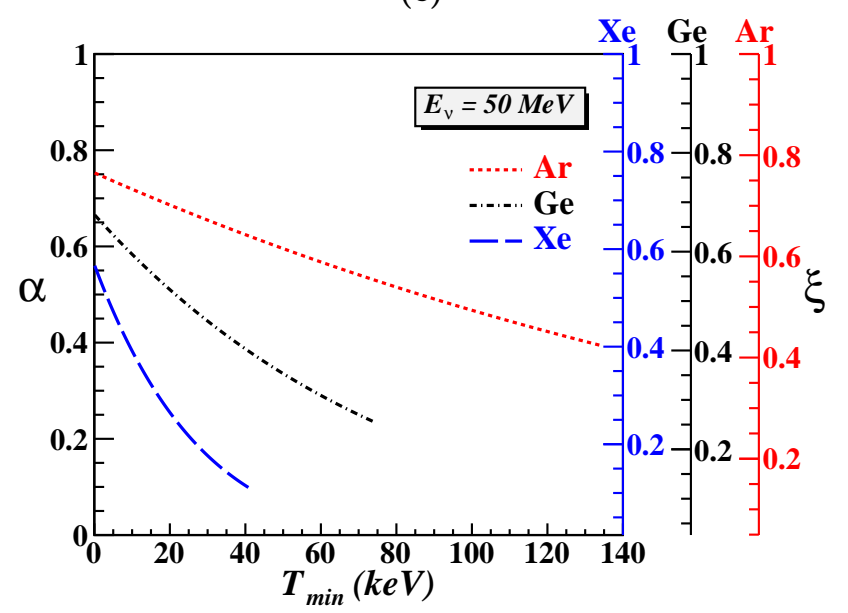

FIG. 2: (a) The $\alpha$-contours on the $\left(N, E_{\nu}\right)$ plane at $T_{\min }=0$, with bands of realistic neutrino sources and target nuclei superimposed. Variations of $\alpha$ and $\xi$ for Ar, Ge,Xe as functions of (b) $E_{\nu}$ at $T_{\min }=0$, and (c) $T_{\min }$ at $E_{\nu}=50 \mathrm{MeV}$, where the end-points correspond to maximum recoil energies. 
(a)

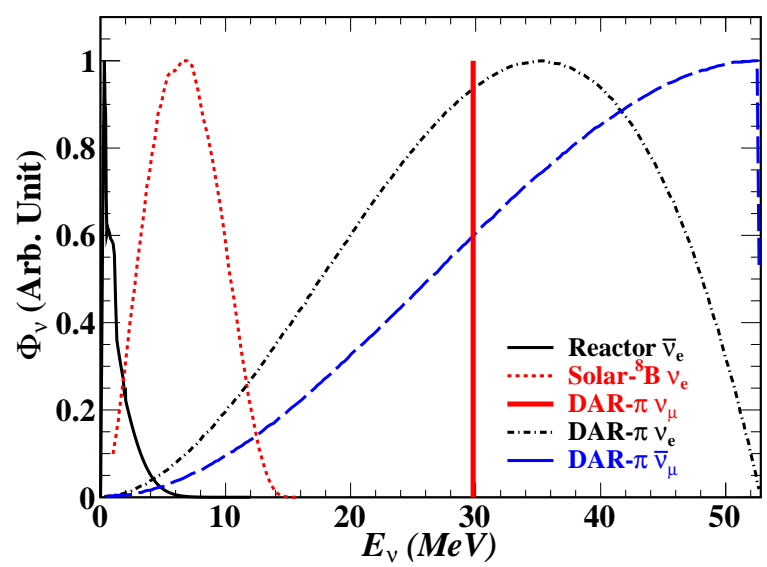

(b)

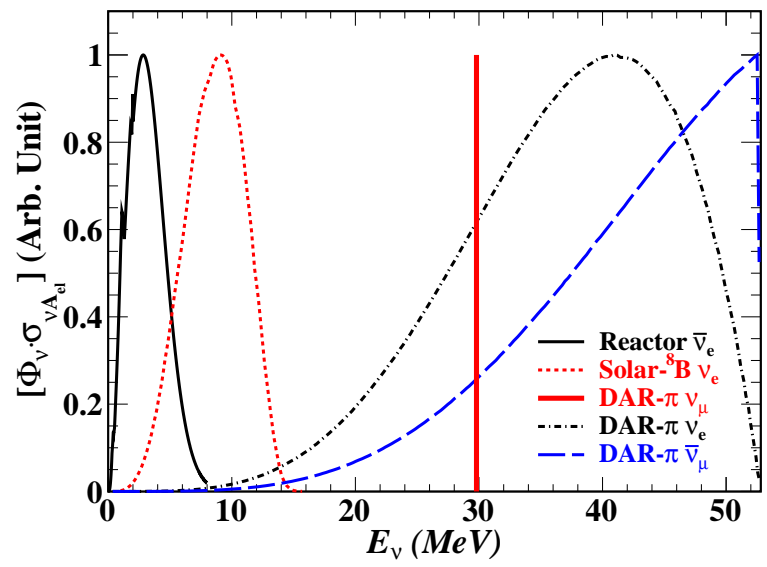

FIG. 3: (a) Neutrino spectra $\left(\Phi_{\nu}\right)$ from reactor $\bar{\nu}_{e}$, DAR- $\pi$ $\left(\nu_{\mu}, \nu_{e}, \bar{\nu}_{\mu}\right)$, and solar- ${ }^{8} \mathrm{~B} \nu_{e}$, normalized by their maxima. (b) Distributions of $\left[\Phi_{\nu} \cdot \sigma_{\nu \mathrm{A}_{e l}}\right]$ at $T_{\min }=0$, which are the weights in the averaging of $(\alpha, \xi)$ to provide measurements of $(\langle\alpha\rangle,\langle\xi\rangle)$.

The relative change in cross-section is an alternative parameter to characterize partial coherency:

$$
\xi \equiv \frac{\sigma_{\nu \mathrm{A}_{e l}}(\alpha)}{\sigma_{\nu \mathrm{A}_{e l}}(\alpha=1)}=\alpha+(1-\alpha)\left[\frac{\left(\varepsilon^{2} Z+N\right)}{(\varepsilon Z-N)^{2}}\right] .
$$

It readily follows that $\xi$ varies linearly with $\alpha$, and both are unity at full coherency.

The parameters $(\alpha, \xi)$ are evaluated with Eqs. $11 \times \sqrt{12}$. respectively, using form factors of Eq. 5 . The $\alpha$-contours on the $\left(N, E_{\nu}\right)$ plane at $T_{\text {min }}=0$ are displayed in Figures $2 \mathrm{a}$, with the bands of realistic neutrino sources and detector target nuclei superimposed. The variations with $E_{\nu}$ at $T_{m i n}=0$ are depicted in Figure $2 \mathrm{~b}$. There is already significant decoherency at $E_{\nu}=50 \mathrm{MeV}$, with values listed in TableI The coherency would further decrease with increasing detector threshold, as illustrated in Figure 2 .

Experimental studies of coherency would be performed with realistic neutrino sources. The current projects are based on reactor $\bar{\nu}_{e}$ [11, DAR- $\pi\left(\nu_{\mu}, \nu_{e}, \bar{\nu}_{\mu}\right)$ [12], as well as the high energy solar- ${ }^{8} \mathrm{~B} \nu_{e}$ in dark matter experiments [10. These neutrino spectra $\left(\Phi_{\nu}\right)$ [12, 15] are (a)

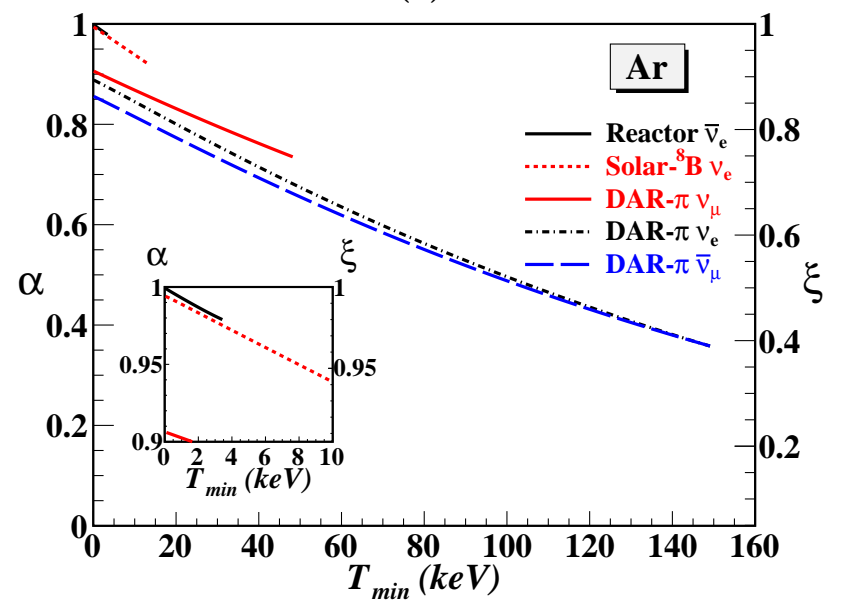

(b)

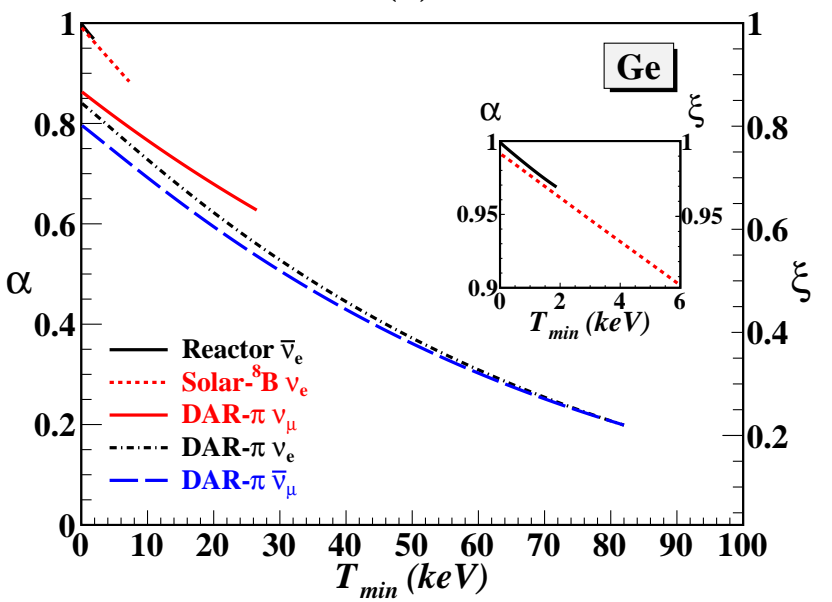

(c)



FIG. 4: Variations of $(\langle\alpha\rangle,\langle\xi\rangle)$ as function of $T_{\min }$ with the reactor $\bar{\nu}_{e}$, solar- ${ }^{8} \mathrm{~B} \nu_{e}$ and DAR- $\pi\left(\nu_{\mu}, \nu_{e}, \bar{\nu}_{\mu}\right)$, for (a) Ar, (b) Ge, and (c) Xe. The end-points correspond to maximum recoil energies allowed by kinematics. 
TABLE II: The half-maxima in the distributions of $\left[\Phi_{\nu} \cdot \sigma_{\nu \mathrm{A}_{e l}}\right]$ at $T_{\text {min }}=0$ for the different neutrino sources, and the values of $\langle\alpha\rangle$ probed by the selected target nuclei. The $\nu_{\mu}$ from DAR- $\pi$ is mono-energetic.

\begin{tabular}{ccccc}
\hline \hline$\nu$ & Half-Maxima of $\left[\Phi_{\nu} \cdot \sigma_{\nu \mathrm{A}_{e l}}\right]$ & \multicolumn{3}{c}{$\langle\alpha\rangle$} \\
Source & in $E_{\nu}(\mathrm{MeV})$ & $\mathrm{Ar}$ & $\mathrm{Ge}$ & $\mathrm{Xe}$ \\
\hline Reactor $\bar{\nu}_{e}$ & $0.96-4.82$ & 1.00 & 1.00 & 1.00 \\
Solar- ${ }^{8} \mathrm{~B} \nu_{e}$ & $5.6-11.9$ & 0.99 & 0.99 & 0.98 \\
DAR- $\pi \nu_{\mu}$ & 29.8 & 0.91 & 0.86 & 0.80 \\
DAR $-\pi \nu_{e}$ & $27.3-49.8$ & 0.89 & 0.83 & 0.76 \\
DAR- $\pi \bar{\nu}_{\mu}$ & $37.5-52.6$ & 0.85 & 0.79 & 0.71 \\
\hline \hline
\end{tabular}

TABLE III: Maximum neutrino energy $\left(E_{\nu}\right)$ with which coherency is maintained among the constituents, as characterized by the parameters $F\left(q_{\max }^{2}\right), \alpha$ and $\xi$ being $>0.95$.

\begin{tabular}{cccc}
\hline \hline Parameter & \multicolumn{3}{c}{ Maximum $E_{\nu}(\mathrm{MeV})$ for } \\
$>0.95$ & $\mathrm{Ar}$ & $\mathrm{Ge}$ & $\mathrm{Xe}$ \\
\hline$F\left(q_{\max }^{2}\right)$ & 17.2 & 14.1 & 11.6 \\
$\alpha$ at $T_{\min }=0$ & 21.1 & 17.4 & 14.3 \\
$\xi$ at $T_{\min }=0$ & 21.6 & 17.6 & 14.4 \\
\hline \hline
\end{tabular}

depicted in Figure 3a. Experiments on $\nu \mathrm{A}_{e l}$ scattering provide measurements of $(\langle\alpha\rangle,\langle\xi\rangle)$, which are averages of $(\alpha, \xi)$ weighted with the distributions of $\left[\Phi_{\nu} \cdot \sigma_{\nu \mathrm{A}_{e l}}\right]$. Those at $T_{m i n}=0$ are displayed in Figure $3 \mathrm{~b}$.

The variations of $(\langle\alpha\rangle,\langle\xi\rangle)$ with detector threshold $T_{\min }$ for the different neutrino sources and targets (Ar, Ge,Xe) are depicted in Figures 4a,b\&c, respectively. The values of $\langle\alpha\rangle$ at $T_{\min }=0$ are summarized in Table II There is strong complementarity between the configurations. The combined measurements of differential crosssections allow studies of the transitions from coherency to decoherency in $\nu \mathrm{A}_{e l}$. In particular the low energy reactor $\bar{\nu}_{e}$ and solar- ${ }^{8} \mathrm{~B} \nu_{e}$ probe the full coherency region $(\alpha>0.9)$, while the intermediate energy DAR- $\pi \nu$ 's allow measurements in the transition regions $(0.9>\alpha>0.1)$.
An objective with the studies of $\nu \mathrm{A}_{e l}$ scattering is to probe physics beyond SM [3, 4]. A direct approach would be to compare the measured cross-sections with the SM predictions given in Eqs. 2 \&44. The sensitivities would be limited by the uncertainties of the form factors in describing the nuclear effects. This favors measurements to be performed at regimes of $F\left(q^{2}\right) \simeq 1$. The maximum $E_{\nu}$ 's which retain $F\left(q_{\max }^{2}\right)>0.95$ are listed in Table III

Another distinctive BSM signature is that $\sigma_{\nu \mathrm{A}_{e l}}$ would no longer vary as $[\varepsilon Z-N]^{2}$ even in the coherency regime of $\alpha \simeq 1$. Dependence characteristics of the deviations can reveal the nature of the new physics couplings. For instance, anomalous neutrino magnetic moments would give rise to an additional contribution which scales as $Z^{2}$ (that is, $\left(\mathcal{X}_{j}, \mathcal{Y}_{k}\right)=(1,0)$ in Eq. 8) [16], while BSM physics giving rise to the dark matter spinindependent couplings are usually taken as varying with $A^{2}$ or $\left(\mathcal{X}_{j}, \mathcal{Y}_{k}\right)=(1,1)$ [10. Sensitivities would be constrained for measurements in kinematics space where coherency is partial. The maximum $E_{\nu}$ at $T_{m i n}=0$ which maintain coherency with $\alpha$ and $\xi$ at $>0.95$ are shown in Table III. Low energy neutrino sources like reactor and solar neutrinos are better suited to probe BSM effects in $\nu \mathrm{A}_{e l}$, although more sensitive experiments are necessary since the measurable recoil energy is lower. These experiments do not have beam-structures for background subtraction but are not vulnerable to neutrino-induced neutron background like those with DAR- $\pi$ sources.

Detailed quantitative studies on the search strategies and potential reaches of different BSM models with $\nu \mathrm{A}_{e l}$ interactions, as well as the sensitivity constraints due to decoherency effects are subjects of future research.

This work is supported by contracts 102-2112-M-002013-MY3, 104-2112-M-001-038-MY3 and 104-2112-M259-004-MY3 from the Ministry of Science and Technology, Taiwan, 2015-ECP4 from the National Center of Theoretical Sciences, Taiwan, 114F374 from TUBITAK, Turkey, and 11475117 from the National Natural Science Foundation, China.
[1] D.Z. Freedman, Phys. Rev. D 9, 1389 (1974); D.Z. Freedman, D.N. Schramm, and D.L. Tubbs. Ann. Rev. Nucl. Part. Sci. 27, 167 (1977).

[2] D.K. Papoulias and T.S. Kosmas, Adv. High Energy Phys. 2015, 763648 (2015).

[3] L.M. Krauss, Phys. Lett. B 269, 407 (1991); J. Barranco, O.G. Miranda and T.I. Rashba, JHEP. 12, 021 (2005); J. Barranco, O. G. Miranda and T. I. Rashba, Phys. Rev. D 76, 073008 (2007).

[4] K. Scholberg, Phys. Rev. D 73, 033005 (2006).

[5] J.R. Wilson, Phys. Rev. Lett. 32, 849 (1974).

[6] K. Patton et al., Phys. Rev. C 86, 024612 (2012).

[7] C.J. Horowitz, K.J. Coakley, and D.N. McKinsey, Phys. Rev. D 68, 023005 (2003).

[8] J. Learned, Nucl. Phys. B (Proc. Suppl.) 143, 152 (2005).

[9] J. Monroe and P. Fisher, Phys. Rev. D 76, 033007 (2007); A. Gütlein et al., Astropart. Phys. 34, 90 (2010);
J. Billard, E. Figueroa-Feliciano, and L. Strigari. Phys. Rev. D 89, 023524 (2014).

[10] M. Drees and G. Gerbier, Review of Particle Physics Chin. Phys. C 38, 353 (2014), and references therein.

[11] H.T. Wong et al., J. Phys. Conf. Ser. 39, 266 (2006). A.K. Soma et al., arXiv:1411.4802 (2014).

[12] F.T. Avignone and Yu.V. Efremenko, J. Phys. G 292615 (2003); D. Akimov et al., arXiv:1509.08702 (2015) and references therein for current experimental projects.

[13] J. Engel, Phys. Lett. B 264, 114 (1991).

[14] See, for example, D. Scully, Ph.D Thesis, U. Warwick, danielscully.co.uk/thesis/thesis.pdf (2013).

[15] H.T. Wong et al., Phys. Rev. D 75, 012001 (2007); G. Bellini et al., Nature 512, 383 (2014)

[16] P. Vogel and J. Engel, Phys. Rev. D 39, 3378 (1989). 